(2) Open Access Full Text Article

\title{
Protective effect of Huang Gan formula in 5/6 nephrectomized rats by depressing the $\mathrm{Wnt} /$ $\beta$-catenin signaling pathway
}

This article was published in the following Dove Press journal:

Drug Design, Development and Therapy

3 June 2015

Number of times this article has been viewed

\author{
Liqian Mo' \\ Xiaoyan Xiao' \\ Shaolian Song' \\ Hui Miao ${ }^{2}$ \\ Shiting Liu' \\ Dan Guo' \\ Xiao $\mathrm{Li}^{3}$ \\ Can $\mathrm{Bu}^{\prime}$ \\ Lianbing Hou' \\ Xixiao Yang' \\ 'Department of Pharmacy, Nanfang \\ Hospital, Southern Medical University, \\ ${ }^{2}$ School of Pharmaceutical Sciences, \\ Sun Yat-sen University, Guangzhou, \\ ${ }^{3}$ Department of Pharmacy, The \\ Affiliated Hospital of Qingdao \\ University Medical School, Qingdao, \\ People's Republic of China
}

Correspondences: Lianbing Hou;

Xixiao Yang

Department of Pharmacy, Nanfang Hospital, Southern Medical University, No 1838, Guangzhou Boulevard (North), Guangzhou 5105I5, People's Republic of China

Tel +86 $2061642175 ;+862062787215$

$\mathrm{Fax}+862087701797$

Email hlianbing@163.com; yxixiao@ hotmail.com

\begin{abstract}
Huang Gan formula (HGF) is a new traditional Chinese herbal medicine created according to the basic theory of traditional Chinese medicine. The aim of this study is to evaluate the effects of HGF on chronic kidney disease and determine the mechanisms of action. The extract of HGF was prepared, and qualitative and quantitative determination of phytochemical was performed with quadrupole time-of-flight mass spectrometer and high-performance liquid chromatography. Sprague-Dawley rats $(n=72)$ were submitted to $5 / 6$ nephrectomy $(N x)$, and then respectively treated with uremic clearance granule, losartan, HGF low dose, HGF middle dose, and HGF high dose once per day for 12 weeks. The sham group of operated rats $(n=22)$ was treated with normal saline or HGF middle dose as a background control group. Blood and urine biochemical parameters, renal tissue morphology, and mRNA and proteins of Wnt/ $\beta$-catenin signaling pathways were investigated. The results showed that the quality of the extraction process could be controlled, and a total of eight major compounds were identified and quantified. HGF could decrease the level of serum creatinine, blood urea nitrogen, and urine protein and increase the renal index and creatinine clearance rate in a dose-dependent manner. HGF also remarkably reduced the glomerulosclerosis and tubulointerstitial fibrosis by blocking the Wnt/ $\beta$-catenin signaling pathway through inhibiting the Wnt1, $\beta$-catenin, transcription factor 4 , and fibronectin 1 expressions, simultaneously measured through mRNA and protein levels in the remnant kidney. These results suggest that extraction of HGF could improve remnant renal function and possibly ameliorate glomerulosclerosis and tubulointerstitial fibrosis by depressing the $\mathrm{Wnt} / \beta$-catenin signaling pathway.
\end{abstract}

Keywords: Huang Gan formula, traditional Chinese medicine, chronic kidney disease, Wnt/ $\beta$-catenin signaling pathway

\section{Introduction}

Chronic kidney disease (CKD) was defined in The National Kidney Foundation Kidney Disease Outcomes Quality Initiative (KDOQI) clinical practice guidelines as kidney damage or glomerular filtration rate (GFR) lower than $60 \mathrm{~mL} / \mathrm{min}$ per $1.73 \mathrm{~m}^{2}$ of tissue for 3 months or longer. ${ }^{1}$ Prevalence of CKD is estimated to be $8 \%-16 \%$ worldwide, and its incidence is increasing in developed and developing nations. ${ }^{2} \mathrm{CKD}$ has become a major public health problem on a global scale because of its serious complications and poor prognosis, and it presents huge socioeconomic burdens to patients, families, and societies. ${ }^{3}$

There are many risk factors leading to $\mathrm{CKD}$, including hypertension, diabetes mellitus, obesity, infections, xenobiotics, herbal or environmental toxins, mechanical obstruction, and deposition of immune complexes. ${ }^{2,4}$ Despite the different factors leading to CKD, the kidney will eventually exhibit the same pathologic characteristics, including glomerulosclerosis and tubulointerstitial fibrosis, which show main 
histopathological features such as myofibroblast proliferation and excessive accumulation of extracellular matrix (ECM). ${ }^{5}$ Molecular mediators involved with fibrogenic pathway include transforming growth factor (TGF- $\beta$ ), bone morphogenic protein, connective tissue growth factor, plateletderived growth factor, etc. These pathways represent new potential targets for novel strategies to inhibit or retard the progress of kidney fibrosis. ${ }^{5,6}$

The Wnt/ $\beta$-catenin signaling pathway is the canonical pathway, was recently discovered and plays an important role in mediating the renal fibrosis. ${ }^{7,8}$ In the absence of the Wnt ligand, $\beta$-catenin is bound to the scaffold proteins axis inhibition protein (Axin) and adenomatous polyposis coli, and then glycogen synthase kinase (GSK)-3 $\beta$ is phosphorylated to form a degradation complex, which mainly degrades $\beta$-catenin to prevent entry into the nucleus. Upon Wnt activation, Wnt ligands bind to and activate two distinct cell surface receptors named frizzled and low density lipoprotein receptor-related protein (LRP) 5 or 6 and induce the phosphorylation of LRP6, which recruits Axin to the plasma membrane, resulting in the inhibition of $\beta$-catenin phosphorylation/degradation. Accumulated $\beta$-catenin translocates to the nucleus and complexes with transcription factor/lymphoid enhancer factor (TCF/LEF), moderating the canonical Wnt-target gene transcription. ${ }^{9,10}$ A simplified schematic of the pathway is shown in Figure S1. General effects of canonical Wnt-pathway activation include the moderation of cell proliferation, differentiation, and cell fate determination. The different Wnt regulators and regulatory mechanism of Wnt signaling pathway have previously been described (www.stanford.edu/ rnusse/ wntwindow.html). Hyperactive Wnt signaling pathway may lead to the development and progression of renal tubular and glomerular diseases and eventually result in CKD. ${ }^{6-8}$ Thus, the Wnt/ $\beta$-catenin signaling pathway may be a potential and novel target for therapeutic intervention of CKD.

Uremic clearance granule (UCG) was developed by our department, which was the first Chinese herbal medicine in the field on prevention of CKD progress in People's Republic of China. Because of the problems and deficiencies in the production and promotion of UCG, our research group intended to work for the secondary development and simplify the prescription according to the theoretical basis of traditional Chinese medicine (TCM), and eventually got the new production called Huang Gan formula (HGF), which had been demonstrated could significantly decrease the serum creatinine (Scr) and blood urea nitrogen (BUN), inhibit oxidative stress in CKD rat models induced by adenine, and delay the progress of renal fibrosis. In this study, we intend to use high-performance liquid chromatography (HPLC)quadrupole time-of-flight mass spectrometer (Q-TOF-MS) techniques to identify and detect the main ingredient and content of HGF. Based on identified effective substance, the therapeutic effect of HGF then would be evaluated by prepared $5 / 6 \mathrm{Nx}$ rat model of CKD. Finally, we investigate the changes in $\mathrm{Wnt} / \beta$-catenin signaling pathway in renal, to explore the possible anti-renal fibrosis mechanisms of HGF.

\section{Materials and reagents}

The herbs of rheum, Radix Bupleuri, Rhizoma Zingiberis, Radix Glycyrrhizae, and Radix Aconiti Lateralis Preparata, were purchased from Kangmei Pharmaceutical Co, Ltd (Guangdong, People's Republic of China) and authenticated by Professor Hongwei Zhang of School of Traditional Chinese Medicine in Southern Medical University. Losartan was purchased from Merck \& Co., Inc. (Whitehouse Station, NJ, USA), and the UCG was purchased from Consun Pharmaceutical Group Limited (Guangzhou, People's Republic of China). Both the one-step reverse transcriptase-polymerase chain reaction (RT-PCR) kit (DRR036A) and the SYBR Premix Taq kit (DRR820A) for real-time PCR were obtained from TaKaRa Biotech Co. (Dalian, People's Republic of China). Rabbit polyclonal antibody Wnt1 (ab85060; Abcam, Inc., Cambridge, MA, USA), $\beta$-catenin (BS1982; Bioworld Technology, Inc., St Louis Park, MN, USA), GSK-3 $\beta$ (sc9166; Santa Cruz Biotechnology, Santa Cruz, CA, USA), p-GSK-3 $\beta$ (sc-135653; Santa Cruz Biotechnology Inc., Dallas, TX, USA), transcription factor 4 (TCF4) (BS6172; Bioworld Technology, Inc.), dickkopf 1 (Dkk1) (BS7731; Bioworld Technology, Inc.), fibronectin 1 (Fn1) (ab2413; Abcam, Inc.), and glyceraldehyde 3-phosphate dehydrogenase (GAPDH) (ab9485; Abcam, Inc.) were purchased. Reference substances such as liquiritin, tectoridin, luteolin, aloe-emodin, rhein, emodin, chrysophanol, and physcion were provided by National Institutes for Food and Drug Control (Beijing, People's Republic of China).

\section{Extract preparation}

Radix et Rhizoma Rhei (307.1 g) and Radix Bupleuri $(614.3 \mathrm{~g})$ were macerated with $70 \%$ ethanol $(1: 7, \mathrm{~m} / \mathrm{v})$ for 2 hours and then extracted by refluxing for a period of 1.5 hours for three times. Rhizoma Zingiberis (229.9 g), Radix Glycyrrhizae (307.1 g), and Aconiti Lateralis Radix Preparata $(245.7 \mathrm{~g})$ were macerated with water $(1: 7, \mathrm{~m} / \mathrm{v})$ for 2 hours and decocted twice every 2 hours each time. Two-time water decoction was blended together, filtered with three-layer gauzes, and concentrated to $200 \mathrm{~mL}$. The concentrated 
solution was precipitated with a final concentration of $80 \%$ ethanol for 24 hours. The supernatant merged with the alcohol extraction of Radix et Rhizoma Rhei and Radix Bupleuri for rotary evaporation concentration. The extraction process was performed ten times repeatedly according to the above methods. The yield of extract reached $21.7 \%(\mathrm{~m} / \mathrm{m})$. Similarity indices of the HPLC chromatograms of ten batches of extracts were evaluated by the professional software, Similarity Evaluation System for Chromatographic Fingerprint of Traditional Chinese Medicine (Version 2004A), which was recommended by Chinese Pharmacopoeia Commission and State Food and Drug Administration. Concentrated extract was placed in $4^{\circ} \mathrm{C}$ for use.

\section{Phytochemical analyses}

In all, $50 \mathrm{mg}$ vacuum drying HGF was dissolved in $50 \mathrm{~mL}$ acetonitrile/water $(1: 1, \mathrm{v} / \mathrm{v})$ and sonicated for 15 minutes. The solution was filtered through a $0.45 \mu \mathrm{m}$ nylon filter membrane, and then $20 \mu \mathrm{L}$ samples were submitted for HPLC analysis (LC-20A; Shimadzu, Tokyo, Japan). The reference substance stock solutions $(0.1 \mathrm{mg} / \mathrm{mL})$ were prepared in acetonitrile and used for identification and creation of a standard curve. HPLC with PDA detector was performed to scan the maximum absorption wavelength of HGF samples.

HPLC conditions are as follows: column, ZORBAX

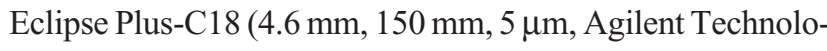
gies, Santa Clara, CA, USA); mobile phase, consisted of water phase $\mathrm{A}(0.1 \%$ formic acid $)$ and organic phase $\mathrm{B}$ (acetonitrile); column, eluted with a gradient of $85 \%-77 \% \mathrm{~A}$ at the beginning of 5 minutes, $77 \%-70 \% \mathrm{~A}$ at 5-30 minutes, $70 \%-55 \% \mathrm{~A}$ at $30-40$ minutes, $55 \%-50 \% \mathrm{~A}$ at $40-60$ minutes, $50 \%-30 \% \mathrm{~A}$ at $60-70$ minutes, $30 \%-27 \%$ A at $70-85$ minutes, $27 \%-20 \%$ $\mathrm{A}$ at $85-90$ minutes; flow rate, $0.8 \mathrm{~mL} / \mathrm{min}$; column temperature, $30^{\circ} \mathrm{C}$; and detection, $254 \mathrm{~nm}$.

The HPLC-Q-TOF-MS spectrometry was performed for qualitative research. The operating parameters for the electrospray ion (ESI) were as follows: the capillary voltage for positive mode (ESI+) and negative mode (ESI-) ionization was set to $\pm 1.59 \mathrm{kV}$. Drying gas $\left(\mathrm{N}_{2}\right)$ flow rate was set to $1.5 \mathrm{~L} / \mathrm{min}$ at a temperature of $200^{\circ} \mathrm{C}$. The collision energy was set at $15-40 \mathrm{~V}$. The LC-MS accurate mass spectra were recorded in the $100-1,000 \mathrm{~m} / \mathrm{z}$ range.

\section{Animals and treatment}

All animal procedures were approved by the Animal Experiment Committee of Southern Medical University. Male 12-week-old Sprague-Dawley rats $(n=94)$ weighing 180-200 g were purchased from the Southern Medical
University Animal Experiment Center. All animals were housed in an environmentally controlled room at $24^{\circ} \mathrm{C} \pm 2{ }^{\circ} \mathrm{C}$ with a relative humidity of $55 \% \pm 5 \%$ and a 12 -hour light/dark cycle. Food and water were available ad libitum.

After 1 week of adjustment, 5/6 Nx were performed according to the technique described previously ${ }^{11}$ under anesthesia with $10 \%$ chloral hydrate ( $1 \mathrm{~mL} / 300 \mathrm{~g}$ weight). In all, 72 rats were taken for surgical resection of two-third of the left kidney and the remaining 22 rats had a sham operation performed. One week later, the survival ones among the 72 rats were subjected to surgical removal of the right kidney and the sham-operated rats to an identical procedure but without removal of any part of the right kidney. One week after the operation, the surviving $5 / 6 \mathrm{Nx}$ rats $(\mathrm{n}=68)$ were randomized by the percent of the remnant kidney weight removed (right kidney weight - weight of two poles of left kidney)/(right kidney weight $\times 100)$, divided into six subgroups $(n=11-12$ in each subgroup). The sham-operated rats $(n=22)$ were randomly divided into the sham-operated group (Sham) and the background control group (BC group).

The dose administration was calculated according to the rat body surface area compared to human. After 1 week of acclimatization, Scr and BUN levels were determined, which indicated that the 5/6 Nx model was successful if the levels were double the sham group. Then, intragastric administration was performed in each group as follows: losartan group (losartan; $30 \mathrm{mg} / \mathrm{kg} /$ day), UCG (3.6 g/kg/day), HGF low dose (HGF-L; $3.6 \mathrm{~g} / \mathrm{kg} /$ day, clinical equivalency dose) group, HGF middle dose (HGF-M; $7.2 \mathrm{~g} / \mathrm{kg} /$ day) group, and HGF high dose (HGF-H; $14.4 \mathrm{~g} / \mathrm{kg} /$ day) group. BC group was given HGF-M $(7.2 \mathrm{~g} / \mathrm{kg} /$ day $)$, while the sham and 5/6 Nx groups were given the same volume of saline solution. All animals were treated by oral gavage every 12 hours continuously for 12 weeks.

\section{Sample collection and biochemical marker detection}

After 12 weeks of treatment, each rat was fasted and placed in an individual metabolism cage for 24 hours urine collection. The serum was collected from abdominal aortic arterial puncture while under anesthesia. The levels of the Scr, BUN, urine creatinine (Ucr), and urine protein (UPr) were detected using an automatic biochemical analyzer (Olympus AU5421; Olympus Corporation, Shinjuku, Tokyo, Japan). The remnant renal tissue was divided into three parts after weighing. One-third was fixed in 10\% neutral-buffered formalin for histopathological and immunohistochemistry examination, while the other two-third of the cortex (100 mg each) was stored at $-80^{\circ} \mathrm{C}$ for RT-PCR and western blot (WB). 


\section{Histology and immunohisto- chemistry staining}

The fixed renal tissues underwent conventional alcohol dehydration, were embedded in paraffin and took serial sections ( $4 \mu \mathrm{m}$ for each section), stained with hematoxylin-eosin (HE) and Masson's trichrome, and examined under a light microscope (Olympus). A semi-quantitative analysis of optical density was performed using Image-Pro Plus 6.0 image system (Media Cybernetics, Inc., MD, USA) for Masson staining pictures with the following conditions: ten non-overlapping renal interstitial vision under $400 \times$ magnification. The semiquantitative analysis was determined by the percentage of the positive staining area accounted for over the entire visual field. All measurements were performed in a blinded fashion.

Immunohistochemical staining was performed using routine protocol. ${ }^{12}$ The sections were stained with the following rabbit polyclonal antibodies: Wnt1, $\beta$-catenin, GSK-3 $\beta$, TCF4, Dkk1, and Fn1. The IgG isotype negative control was stained by replacing the primary antibody with rabbit serum. The average optical density values were semi-quantified by Image-Pro Plus 6.0 under $400 \times$ magnification.

\section{Real-time quantitative PCR}

Total RNA was extracted from the renal cortex tissue $(n=6)$ using TRIzol RNA isolation system (Thermo Fisher Scientific, Waltham, MA, USA). The cDNA was synthesized, and PCR was carried out using a standard PCR kit. PCR primers are shown in Table S1. RT-PCR was performed using LightCycler $^{\circledR} 480$ (Hoffmann-La Roche Ltd., Basel, Switzerland), which expresses the number of threshold cycle $\left(C_{t}\right)$. Relative expression levels of target genes were determined by calculating $2^{-\mathrm{DDC} C_{.} \cdot{ }^{13}}$

\section{WB analysis}

Western blotting was conducted as previously described. ${ }^{14}$ Proteins were extracted from approximately $100 \mathrm{mg}$ renal cortex tissues and quantified with bicinchoninic acid assay kit (KeyGen Biotech, Nanjing, People's Republic of China). The proteins of six randomly selected samples were mixed in equal amounts, and total protein was analyzed in triplicates. Antibodies used were the same as the immunohistochemical staining kit, except for internal control GAPDH.

\section{Statistical analyses}

Data are presented as the mean \pm standard deviation. Statistical analyses were performed using one-way analysis of variance (SPSS13.0, Chicago, USA) test followed by Tamhane's test to compare three or more groups or by Student's $t$-test to compare two groups. $P<0.05$ was considered to be statistically significant.

\section{Results \\ Quality control and phytochemical analyses}

Figure 1 shows the HPLC fingerprint chromatogram of ten different batches of HGF extract. The standardized characteristic chromatogram was generated by the similarity evaluation system according to the ten chromatograms (shown in Supplementary materials). The similarity indices were calculated and compared with the standardized characteristic chromatogram, which was $0.929,0.987,0.982$, $0.965,0.993,0.974,0.994,0.997,0.942$, and 0.981 from S1 to $\mathrm{S} 10$ in sequence. All of the similarity indices were greater than 0.92 , indicating the components of different batches of extracts were similar and stable. The reference fingerprint of HGF (shown in Figure S2) was produced according to HPLC chromatograms of the ten batches of HGF extracts by Similarity Evaluation System for Chromatographic Fingerprint of Traditional Chinese Medicine (Version 2004A). A total of 21 common peaks were marked, and the maximum peak was set to 18 , which was the reference chromatographic peak, while the retention time and peak area were set to 1 . The relative retention time and relative peak area of common peaks compared to peak 18 are listed in Tables S2 and S3. Fingerprint results showed that the ten batches of HGF possessed well consistency and the extraction method was stable. Using HPLC fingerprint can better control overall quality of HGF extract, and the quality of the extraction process could also be controlled.

The HPLC-Q-TOF-MS spectrometry was used to characterize the chemical compositions within the HGF extract. The total ion current chromatograms of the HGF extract is shown in Figure 2. A total of eight major compounds were identified and quantified by comparing reference standards. Their chemical names and contents $(\mathrm{m} / \mathrm{m})$ were liquiritin $(0.167 \%)$, tectoridin (1.98\%), luteolin (1.03\%), aloe-emodin (3.78\%), rhein (3.13\%), emodin (1.54\%), chrysophanol (2.72\%), and physcion (1.55\%). The results of MS qualitative analysis of HGF is shown in Table S4.

\section{Assessment of renal function}

As shown in Table 1, the 5/6 Nx group renal index, Scr, BUN, and UPr levels were significantly higher than the sham and $\mathrm{BC}$ groups, and the creatinine clearance rate $(\mathrm{Ccr})$ was significantly lower. The levels of renal index in medicated 


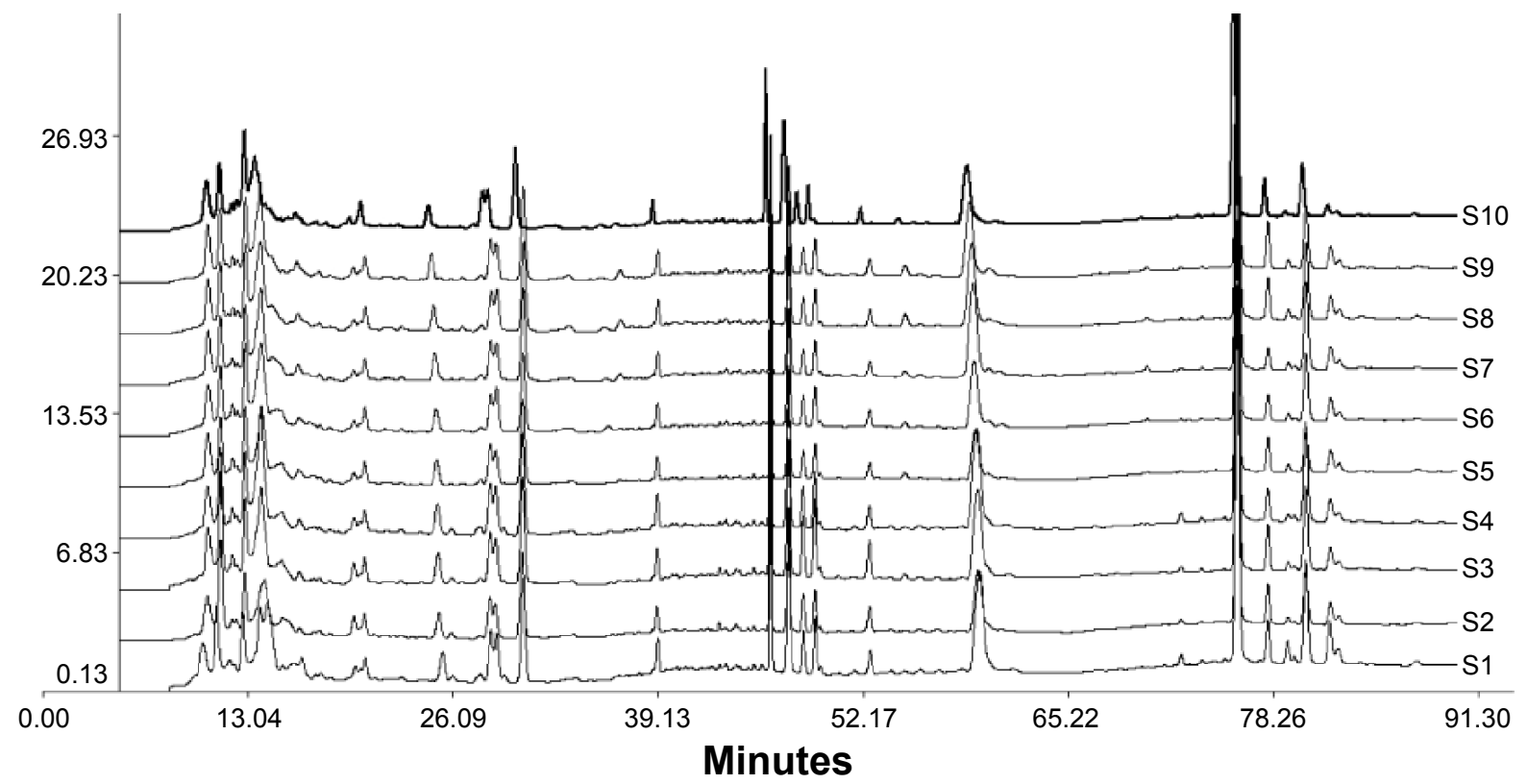

Figure I Fingerprint chromatogram of ten batches of HGF extracts.

Notes: SI-SIO were the UV-HPLC chromatograms of ten batches of HGF extracts, which were detected under the same HPLC conditions.

Abbreviations: HGF, Huang Gan formula; UV, ultraviolet; HPLC, high-performance liquid chromatography.

A

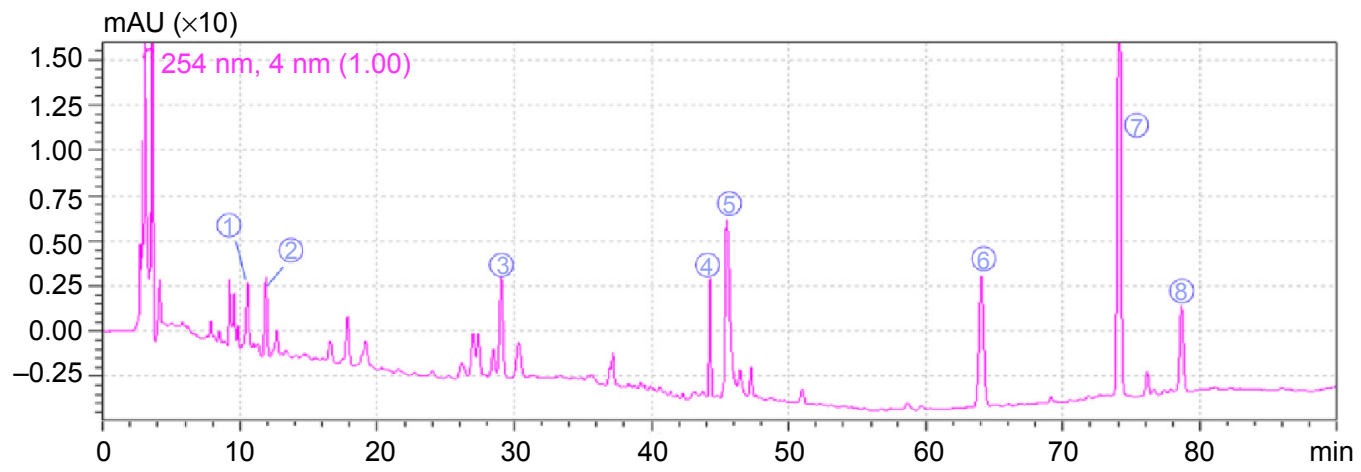

B $\quad(\times 1,000,000)$

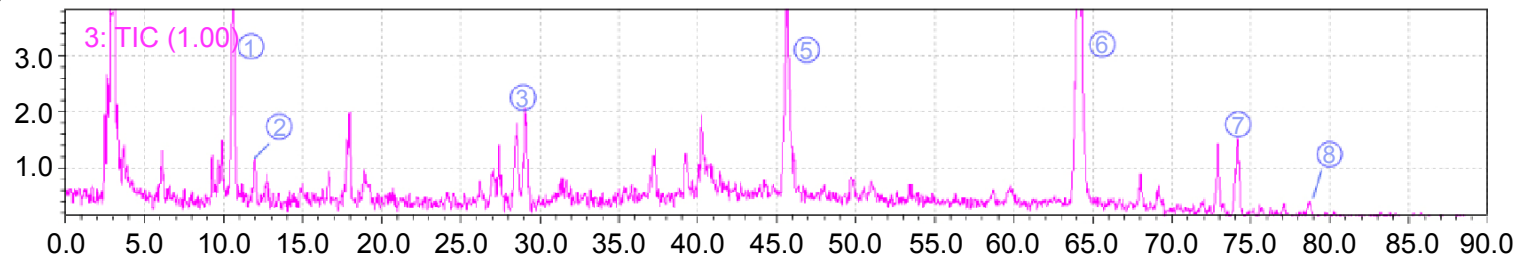

C $(\times 1,000,000)$

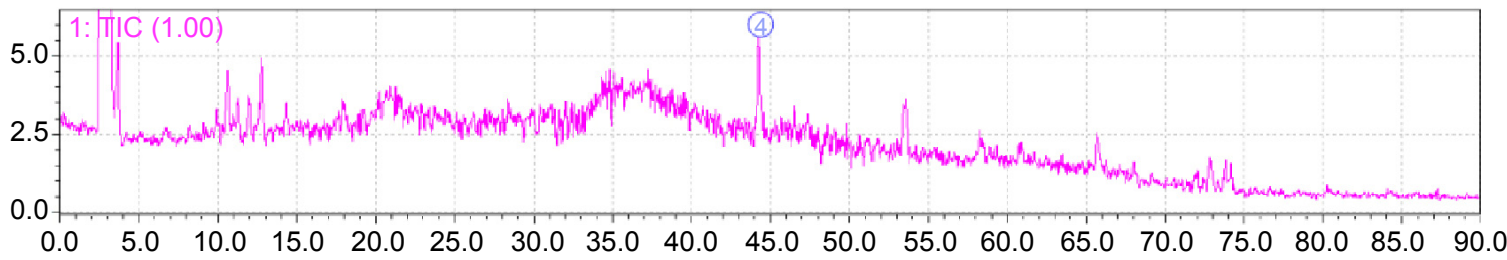

Figure 2 HPLC and Q-TOF-MS chromatograms of HGF extract.

Notes: UV-HPLC/chromatogram $(254 \mathrm{~nm})(\mathbf{A})$; the total ion current in the negative ion mode in MS (B); and the total ion current in the positive ion mode (C). The same numbers between HPLC and MS chromatograms indicate the same substances. As follows: (1) liquiritin, (2) tectoridin, (3) luteolin, (4) aloe-emodin, (5) rhein, (6) emodin, (7) chrysophanol, and (8) physcion.

Abbreviations: HPLC, high-performance liquid chromatography; Q-TOF-MS, quadrupole time-of-flight mass spectrometer; HGF, Huang Gan formula; UV, ultraviolet; min, minute. 
groups, Scr and BUN in HGF-M and HGF-H groups, and UPr among HGF three dosage groups were all decreased significantly compared with the $5 / 6 \mathrm{Nx}$ group, while the level of Ccr increased in HGF-L and HGF-H groups. All medicated groups had no significant difference with each other, except within the levels of BUN, Ccr, and UPr in HGF groups compared with losartan groups. There was no significant difference noted between sham and BC groups for all biochemical markers above.

\section{Renal histopathology analysis}

$\mathrm{HE}$ and Masson staining showed significant glomerulosclerosis and interstitial fibrosis in the $5 / 6 \mathrm{Nx}$ group 12 weeks following 5/6 $\mathrm{Nx}$ and presented proliferation of mesangial cells, tubular atrophy, and infiltration of large numbers of inflammatory cells in renal interstitium. However, these pathological changes were not observed in the sham and $\mathrm{BC}$ groups, and the three HGF groups attenuated these pathological changes to varying degrees (Figure 3, HE/Masson). The semi-quantitative analysis of Masson trichrome staining showed strikingly increased interstitial fibrotic areas in the 5/6 Nx group compared with the sham and BC groups, and HGF groups had remarkably reduced interstitial fibrosis $(P<0.05)$ (Table 2, Masson).

\section{Expression and distribution of Wnt/ $\beta$-catenin signaling-associated proteins by immunohistochemistry}

Figure 4 IHC shows the representative micrographs of Wnt1, $\beta$-catenin, TCF4, GSK-3 $\beta$, Dkk1, and Fn1 staining within the renal cortex sections from the $5 / 6 \mathrm{Nx}$ group (micrographs of other groups not shown). All proteins were rarely expressed in normal kidneys, but were distributed in different regions. Wnt1, TCF4, and Dkk1 were predominantly localized within the glomeruli and renal tubules in the diseased kidneys, while $\beta$-catenin and GSK-3 $\beta$ were mainly in the glomeruli and distal convoluted tubules. Fn1, the fibrosis related factor, was mostly observed in the glomerular mesangial area and tubulointerstitium. To quantify protein expression, semi-quantitative analysis of average optical density values was performed and showed that all of the proteins expressed significantly higher in the 5/6 Nx group than the sham and $\mathrm{BC}$ groups $(P<0.05)$. HGF inhibited the expression of Wnt1, $\beta$-catenin, TCF4, and Fn1 markedly, but had no effect on expression of GSK-3 $\beta$ and Dkk1, which showed no significant difference compared to the $5 / 6 \mathrm{Nx}$ group $(P>0.05)$ (Table 2). Fn1 was also expressed much less in HGF groups, consistent with the Masson staining (Figure 3).

\section{The mRNA and protein expression of $\mathrm{Wnt} / \beta$-catenin signaling by RT-RCR and WB}

To further investigate the quantified expressions of Wnt/ $\beta$-catenin signaling-associated proteins, RT-PCR and WB were performed in the diseased kidney. As shown in Figure 5, the mRNA expressions of Wnt1, $\beta$-catenin, TCF4, GSK-3 $\beta$, Dkk1, and Fn1 in the 5/6 Nx group were increased dramatically when compared with the sham group $(P<0.01)$. UCG, losartan, and HGF inhibited the mRNA expression of Wnt1, $\beta$-catenin, TCF4, and Fn1 significantly. Simultaneously, the HGF-L, HGF-M, and HGF-H groups also showed inhibition in a dose-dependent manner, though had no significant difference compared with UCG and losartan groups. The GSK-3 $\beta$ and Dkk1 levels were reduced in HGF groups, but had no significant difference compared to the $5 / 6 \mathrm{Nx}$ group $(P>0.05)$.

The results of WB corroborated the RT-PCR findings (Figure 6). The expression levels of Wnt1, $\beta$-catenin,

Table I Biochemical markers of each group, $\bar{x} \pm s(\mathrm{n}=9)$

\begin{tabular}{llllll}
\hline Group & RI & Scr $(\mu \mathbf{m o l} / \mathbf{L})$ & BUN $(\mathbf{m m o l} / \mathbf{L})$ & Ccr $(\mathbf{m L} / \mathbf{m i n})$ & UPr $(\mathbf{m g} / \mathbf{2 4} \mathbf{h})$ \\
\hline Sham & $3.23 \pm 0.29$ & $41.4 \pm 6.0$ & $6.9 \pm 1.3$ & $0.57 \pm 0.16$ & $3.3 \pm 1.4$ \\
BC & $3.33 \pm 0.34$ & $43.0 \pm 5.6$ & $7.0 \pm 0.8$ & $0.54 \pm 0.17$ & $3.7 \pm 1.3$ \\
$5 / 6 \mathrm{Nx}$ & $3.77 \pm 0.44^{\#}$ & $83.0 \pm 5.8^{\#}$ & $15.6 \pm 0.9^{\#}$ & $0.34 \pm 0.12^{\#}$ & $5.1 \pm 0.8^{\#}$ \\
UCG & $3.15 \pm 0.29^{*}$ & $76.6 \pm 5.4^{\#}$ & $13.1 \pm 0.9^{\#, *}$ & $0.53 \pm 0.18^{*}$ & $4.1 \pm 1.6$ \\
Losartan & $3.25 \pm 0.19^{*}$ & $65.2 \pm 5.5^{\#, *}$ & $20.2 \pm 1.9^{\#, *}$ & $0.31 \pm 0.1 I^{\#}$ & $2.2 \pm 1.1^{*}$ \\
HGF-L & $3.20 \pm 0.37^{*}$ & $75.5 \pm 10.3^{\#}$ & $13.5 \pm 1.7^{\#, \Delta}$ & $0.55 \pm 0.17^{*, \Delta}$ & $4.2 \pm 1.0^{*, \Delta}$ \\
HGF-M & $3.37 \pm 0.25^{*}$ & $71.6 \pm 6.1^{\#, *}$ & $13.1 \pm 1.0^{\#, *, \Delta}$ & $0.41 \pm 0.21$ & $3.2 \pm 1.3^{*}$ \\
HGF-H & $3.33 \pm 0.18^{*}$ & $60.0 \pm 13.4^{*}$ & $13.2 \pm 1.0^{\#, *, \Delta}$ & $0.57 \pm 0.16^{*, \Delta}$ & $3.8 \pm 0.7^{*, \Delta}$ \\
\hline
\end{tabular}

Notes: Compared with Sham and BC groups, ${ }^{*} P<0.05$; compared with the $5 / 6 \mathrm{Nx}$ group, ${ }^{* P}<0.05$; and compared with the losartan group, ${ }^{\Delta P}<0.05$. RI $=$ renal weight (mg)/ body weight $(\mathrm{g})$. $\mathrm{Ccr}=$ urine creatinine $(\mu \mathrm{mol} / \mathrm{L}) \times$ urine volume per minute $(\mathrm{mL}) / \mathrm{Scr}(\mu \mathrm{mol} / \mathrm{L})$.

Abbreviations: RI, renal index; Scr, serum creatinine; BUN, blood urea nitrogen; Ccr, creatinine clearance rate; UPr, urine protein; Sham, sham-operated group; BC, background control group; Nx, 5/6 nephrectomy; UCG, uremic clearance granule; HGF-L, Huang Gan formula low dose group; HGF-M, Huang Gan formula middle dose group; HGF-H, Huang Gan formula high dose group. 


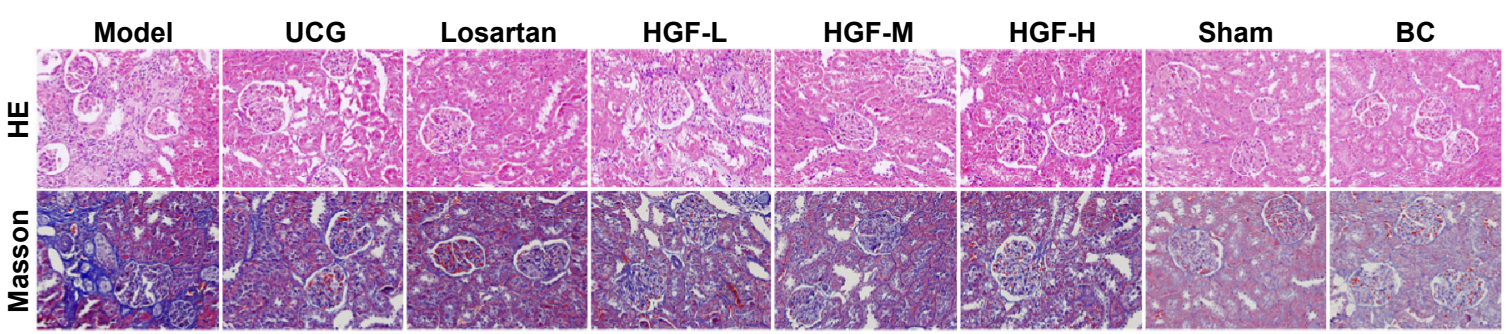

Figure 3 Representative micrographs of HE and Masson staining in the rat renal cortex sections (400x).

Abbreviations: HE, hematoxylin-eosin; UCG, uremic clearance granule; HGF-L, Huang Gan formula low dose group; HGF-M, Huang Gan formula middle dose group; HGF-H, Huang Gan formula high dose group; Sham, sham-operated group; BC, background control group.

TCF4, GSK-3ß, Dkk1, and Fn1 were upregulated in the $5 / 6 \mathrm{Nx}$ group when compared with those in the sham group $(P<0.01)$, while UCG, losartan, and HGF downregulated the expressions of these proteins except for GSK-3 $\beta$ and Dkk1, which as the inhibitors of $\mathrm{Wnt} / \beta$-catenin signaling were both kept at high levels in drug treatment groups, but had no significant difference compared with the $5 / 6 \mathrm{Nx}$ group $(P>0.05)$. The RT-PCR and WB results were approximately consistent with the immunohistochemistry results. All of these indicators showed no significant difference between sham and $\mathrm{BC}$ groups.

\section{Discussion}

The pathogenic mechanisms of CKD are varied, including systemic and glomerular hypertension, excessive expression of TGF- $\beta$, the renin-angiotensin-aldosterone system, podocyte loss, dyslipidemia, etc. ${ }^{5,15}$ However, renal fibrosis is the common end point of virtually all progressive CKDs. Currently, drug treatments of CKD are mainly confined to control renal hypertension and improve the glomerular "high perfusion, high internal pressure and high filtration" state, thereby reducing further lesions of the kidney tissue.
Such drugs used primarily for the angiotensin converting enzyme inhibitors and angiotensin receptor blockers play an important role in control of blood pressure and reduction of proteinuria and TGF- $\beta .{ }^{16}$ Additionally, other treatment strategies, such as lipid-lowering therapy, correction of acidosis, prevention of atherosclerotic events, and modification with low salt and protein diets, have been implemented. But unfortunately, ${ }^{2}$ these therapies only have limited efficacy confronting the complex pathogenic factors and multiple complications of CKD.

UCG as the first and dominant TCM used for treatment of CKD has been employed in People's Republic of China more than a decade, exhibiting positive effects on improving renal function and delaying the progression of chronic renal failure. ${ }^{17,18}$ Losartan, an angiotensin receptor blocker, is recommended by Kidney Disease: Improving Global Outcomes as a pharmacologic therapy for CKD with proteinuria and hypertension. ${ }^{19}$ Both are representative effective drugs commonly used in clinical CKD treatment. We choose these two drugs as the positive control groups, UCG as the Chinese medicine control and losartan as the western medicine control. The present study demonstrated that HGF improved

Table 2 Average optical density values of protein expressions by immunohistochemistry staining in renal tissues of rats in different groups, $\bar{x} \pm s(\mathrm{n}=3)$

\begin{tabular}{|c|c|c|c|c|c|c|c|}
\hline Group & Masson (\%) ${ }^{\nabla}$ & Wntl & $\beta$-catenin & GSK-3 $\beta$ & Dkk I & TCF4 & FnI \\
\hline Sham & $5.1 \pm 0.4$ & $3.31 \pm 0.69$ & $1.05 \pm 0.08$ & $4.0 \pm 0.7$ & $0.42 \pm 0.06$ & $2.5 \pm 0.1$ & $2.60 \pm 0.44$ \\
\hline $\mathrm{BC}$ & $5.3 \pm 0.3$ & $3.37 \pm 0.04$ & $1.45 \pm 0.83$ & $2.7 \pm 0.4$ & $0.35 \pm 0.06$ & $2.2 \pm 0.1$ & $2.90 \pm 0.55$ \\
\hline $5 / 6 \mathrm{Nx}$ & $15.8 \pm 1.4^{\#}$ & $8.92 \pm 0.88^{\#}$ & $4.99 \pm 1.00^{\#}$ & $8.9 \pm 1 . I^{\#}$ & $4.70 \pm 0.12^{\#}$ & $11.2 \pm 1.2^{\#}$ & $6.7 \mathrm{I} \pm 0.38^{\#}$ \\
\hline Losartan & $10.9 \pm 1.7^{\#, *}$ & $8.76 \pm 1.54^{\#}$ & $3.50 \pm 0.35^{\#, *}$ & $8.3 \pm 1.3^{\#}$ & $4.29 \pm 0.70$ & $6.7 \pm 0.3^{\#, *}$ & $5.26 \pm 0.1 I^{\#, *}$ \\
\hline UCG & $9.9 \pm 0.8^{\#, *}$ & $6.70 \pm 0.4 I^{\#, *}$ & $2.54 \pm 0.62^{\#, *}$ & $7.0 \pm 1.4^{\# * *}$ & $3.69 \pm 0.68$ & $4.3 \pm 0.3^{\#, *}$ & $5.36 \pm 0.17^{\#, *}$ \\
\hline HGF-L & $8.1 \pm 0.5^{\#, *, \Delta}$ & $6.34 \pm 0.24^{\#, *, \Delta}$ & $2.97 \pm 0.82^{\#, *}$ & $6.3 \pm 0.5^{\#, *}$ & $3.78 \pm 1.15$ & $6.7 \pm 0.5^{\#, *}$ & $5.56 \pm 0.37^{\# * *}$ \\
\hline HGF-M & $7.3 \pm 0.9^{\#, *, \Delta}$ & $5.95 \pm I .1 I^{\#, *, \Delta}$ & $2.49 \pm 0.57^{\# *}$ & $7.0 \pm 1.4^{\#, *}$ & $4.96 \pm 1.46$ & $6.4 \pm 0.5^{\#, *}$ & $4.72 \pm 0.70^{\#, *}$ \\
\hline HGF-H & $5.6 \pm 1.2^{*, \Delta}$ & $4.32 \pm 0.22^{*, \Delta}$ & $1.83 \pm 0.16^{*, \Delta}$ & $8.8 \pm 0.4^{\#}$ & $6.62 \pm 1.03$ & $6.0 \pm 0.5^{\#, *}$ & 4. $16 \pm 0.49^{\#, *, \Delta}$ \\
\hline
\end{tabular}

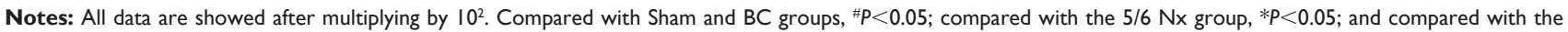
losartan group, ${ }^{\Delta P}<0.05{ }^{\nabla}$, the proportion of positive area.

Abbreviations: GSK, glycogen synthase kinase; DkkI, dickkopf I; TCF4, transcription factor 4; FnI, fibronectin I; Sham, sham-operated group; BC, background control group; Nx, 5/6 nephrectomy; UCG, uremic clearance granule; HGF-L, Huang Gan formula low dose group; HGF-M, Huang Gan formula middle dose group; HGF-H, Huang Gan formula high dose group. 

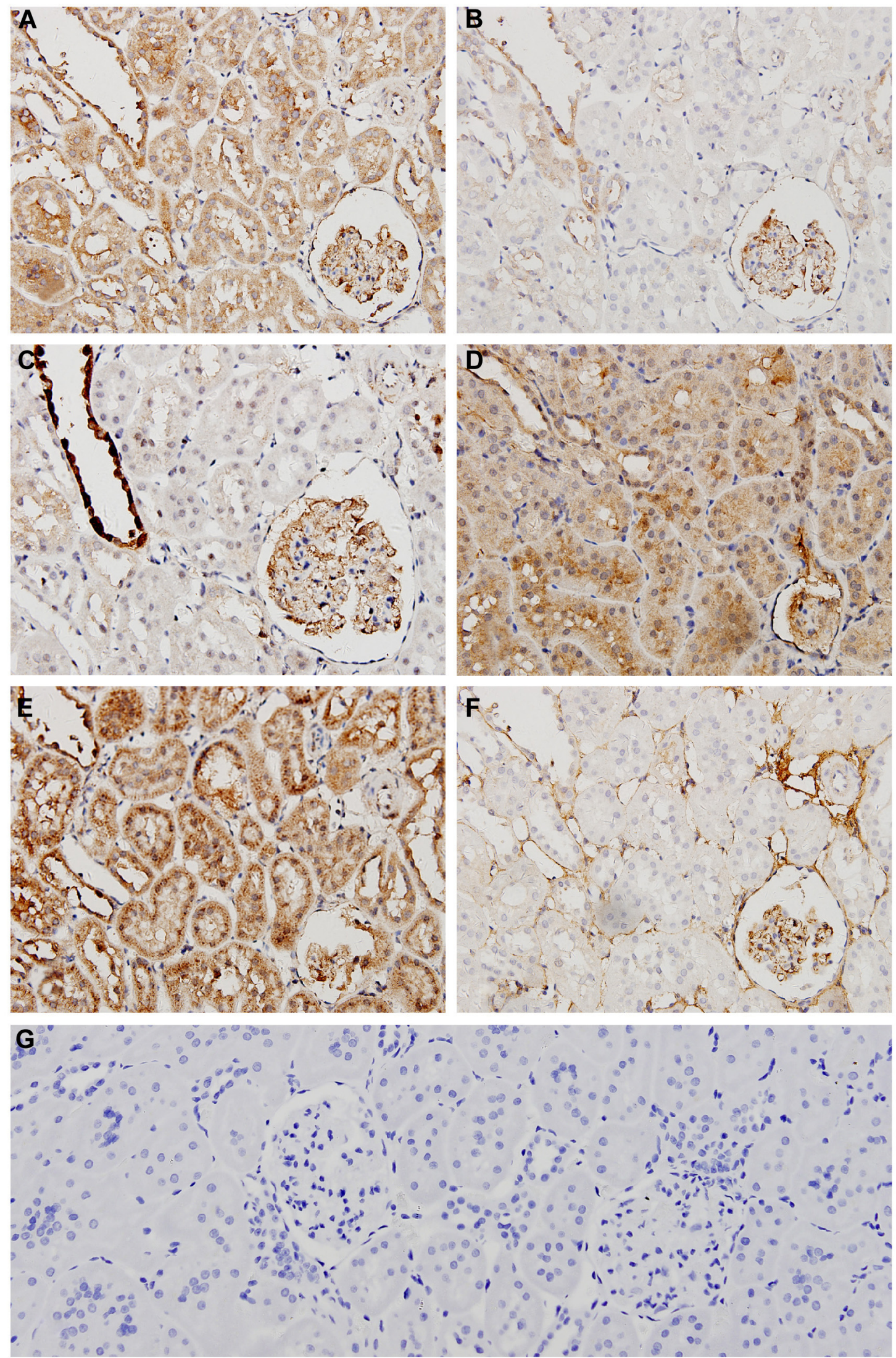

Figure 4 Representative micrographs of immunohistochemistry staining in the $5 / 6 \mathrm{Nx}$ group rat renal cortex sections (400x).

Notes: Immunohistochemistry staining of $5 / 6 \mathrm{Nx}$ group for Wntl (A), $\beta$-catenin (B), GSK-3 $\beta$ (C), DkkI (D), TCF4 (E), FnI (F), and IgG negative control (G),

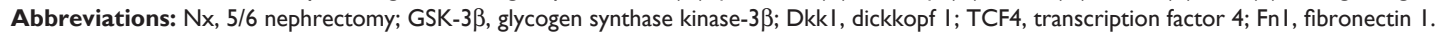



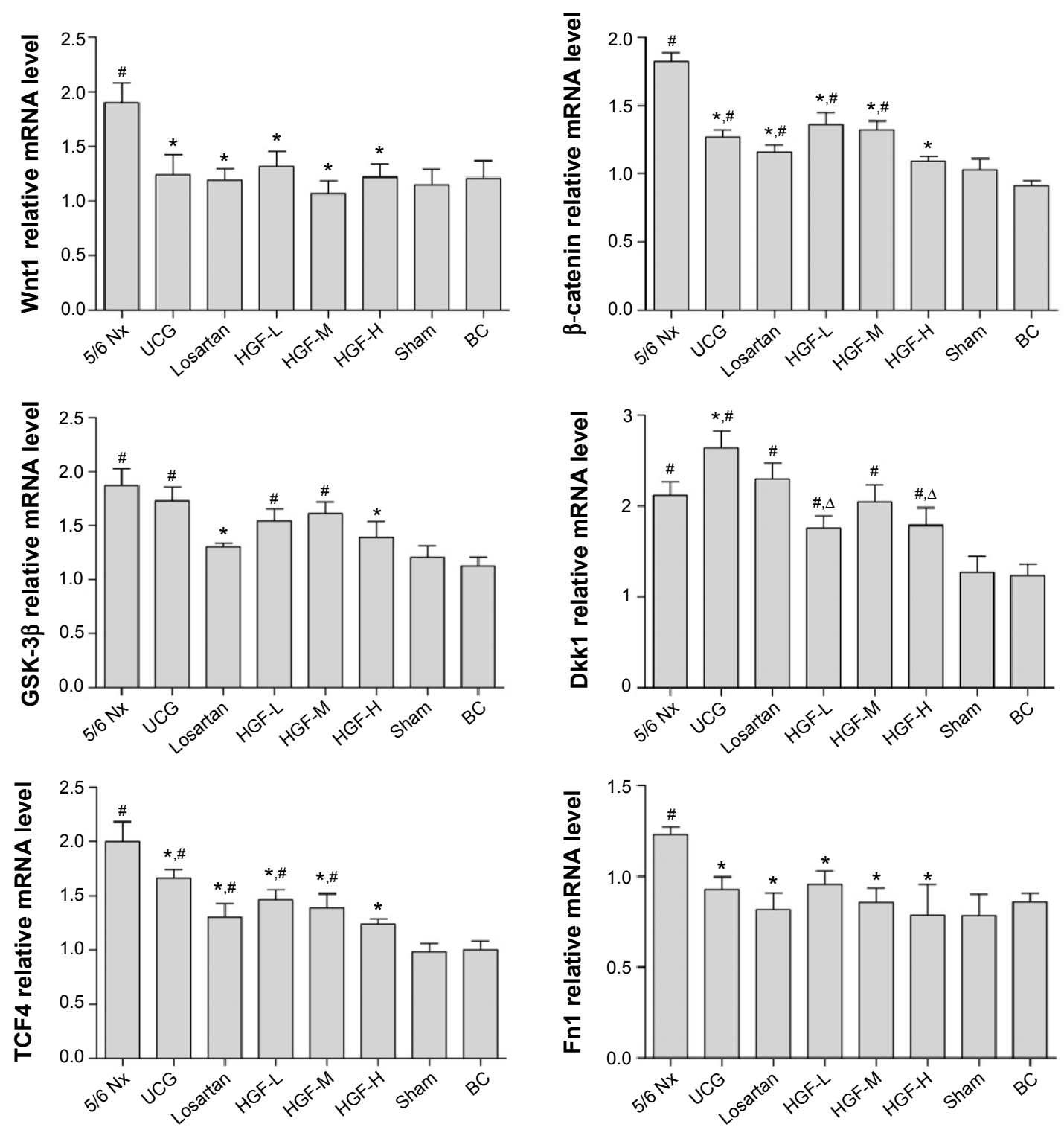

Figure $5 \mathrm{Wntl}, \beta$-catenin, TCF4, GSK-3 $\beta$, Dkkl, and FnI mRNA expression in renal tissues $(\mathrm{n}=6)$.

Notes: Compared with the 5/6 Nx group, ${ }^{*} P<0.05$; compared with Sham and BC groups, ${ }^{\#} P<0.05$; and compared with the losartan group, ${ }^{\Delta P}<0.05$.

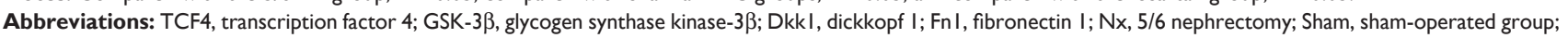
BC, background control group; UCG, uremic clearance granule; HGF-L, Huang Gan formula low dose group; HGF-M, Huang Gan formula middle dose group; HGF-H, Huang Gan formula high dose group.

remnant renal function and alleviated glomerulosclerosis and tubulointerstitial fibrosis showing similar efficiency with UCG. The level of BUN in HGF groups was significantly lower than losartan and the Ccr was much higher than that, which demonstrated that the HGF showed better renal protective effect than losartan. But losartan was the best in decreasing the UPr, which had been confirmed in the published literature. BC group, which has no excision in any part of renal, was given HGF-M. The goals while setting this negative control group were first to evaluate its toxic effects and second to exclude the inflammation, stress reaction, or renal damage induced by HGF self, which may lead to the false negative results. The research results showed that the biochemical and pathological indicators of the $\mathrm{BC}$ group had no significant difference with the sham group, which fitted the expected goal and demonstrated that HGF had no obvious toxic effect on the experimental rats.

TCM formulas often contain multiple medicinal herbs that lead to the action on multiple targets and thereby produce an overall systemic effect. ${ }^{20}$ TCM based on unique theories and long-term practical experiences is very popular in People's Republic of China, especially in delaying renal disease progression and protecting renal function. It has become an indispensable means of treatment of CKD. ${ }^{21}$ 
A

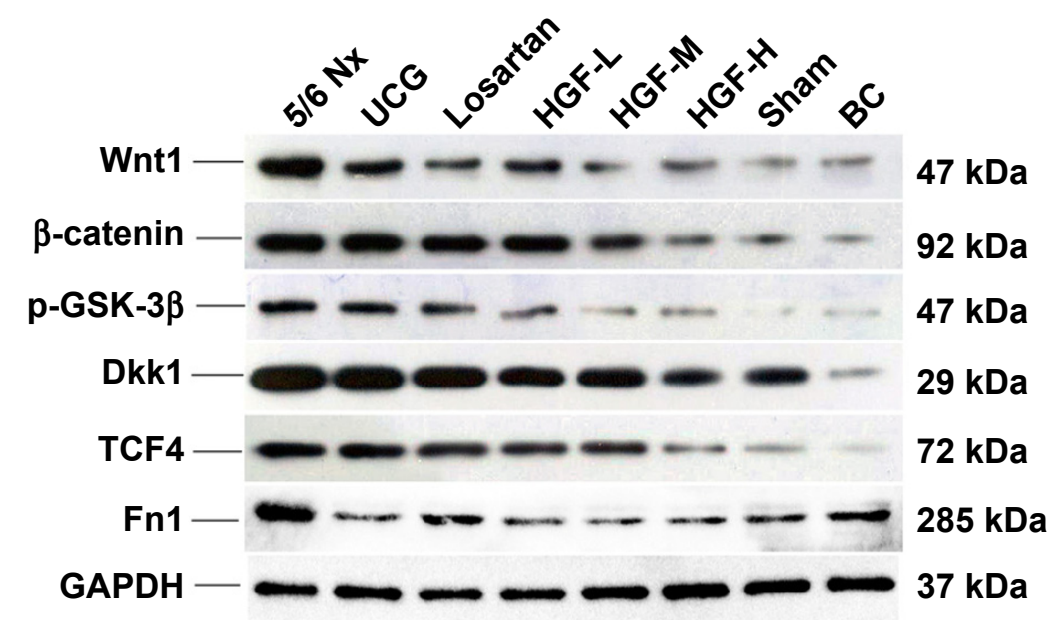

B

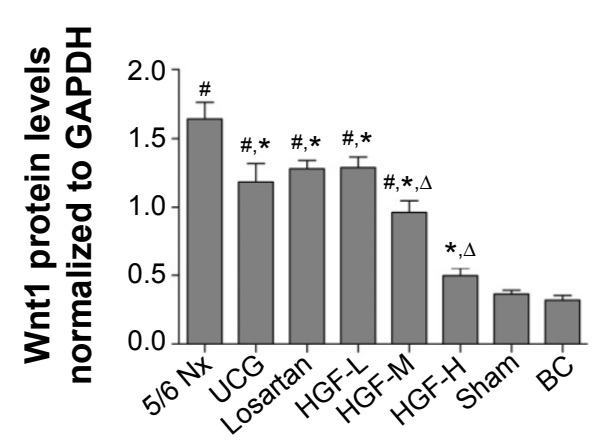

D

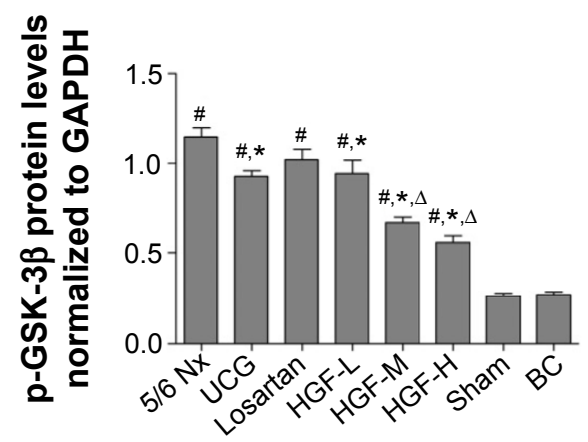

F

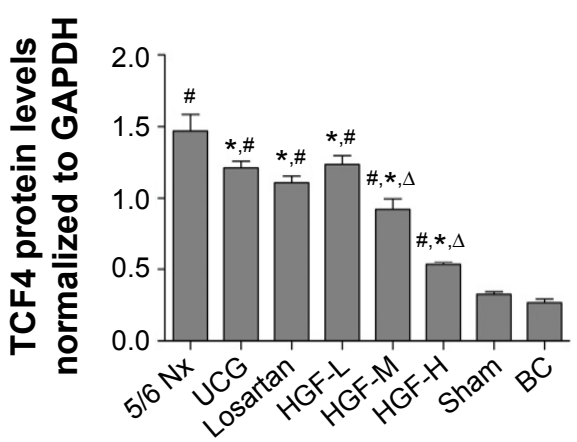

C

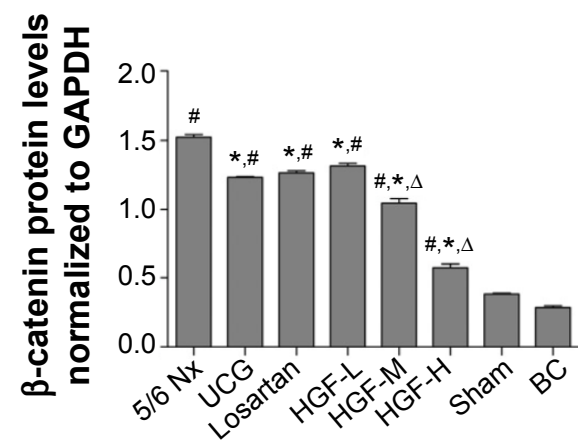

E

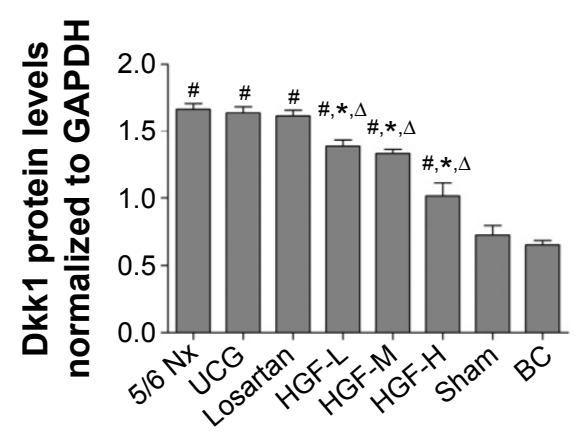

G

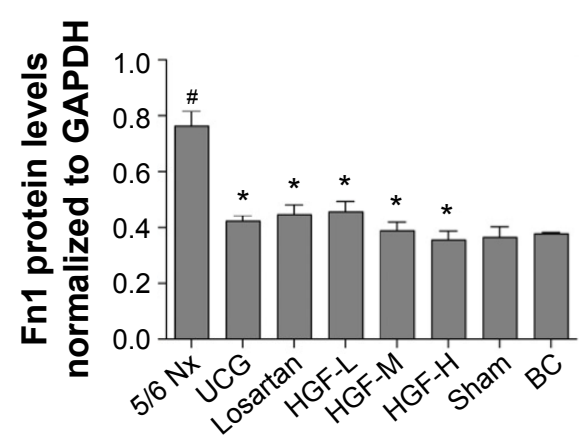

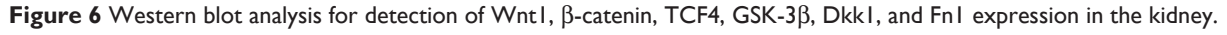

Notes: Western blot bands of different proteins (A); semi-quantitative analysis of the bands (from (B) to (G) are Wntl, $\beta$-catenin, GSK-3ß, DkkI, TCF4, and FnI in sequence). Compared with $5 / 6 \mathrm{Nx}$ group, ${ }^{* P}<0.05$; compared with Sham and $B C$ groups, ${ }^{\# P}<0.05$; and compared with the losartan group ${ }^{\Delta P}<0.05$.

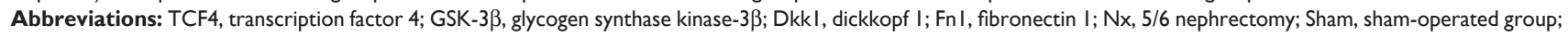
BC, background control group; UCG, uremic clearance granule; HGF-L, Huang Gan formula low dose group; HGF-M, Huang Gan formula middle dose group; HGF-H, Huang Gan formula high dose group; GAPDH, glyceraldehyde 3-phosphate dehydrogenase. 
Many studies have reported the prescriptions of a single herb, decoctions of multiple herbs, or patent medicines that have been used to treat patients with CKD, which exhibit effects including promotion of diuresis, reduction of proteinuria, and improvement of renal function. ${ }^{22}$ The results of this study indicating the components and content of HGF are stable and can be controlled, and the main components are aloe-emodin, rhein, and chrysophanol extracted from rheum. The efficacy of most of these constituents has been verified in vitro and vivo. Similar to emodin, it can reduce glucoseinduced ECM synthesis in human peritoneal mesothelial cells. ${ }^{23}$ Rhein therapy markedly ameliorated renal interstitial fibrotic lesions, and the primary target was TGF- $\beta 1 .{ }^{24}$ In this study, we also proved that HGF could decrease the level of Scr, BUN and UPr and ameliorate tubulointerstitial fibrosis in $\mathrm{CKD}$ rats, which may act through the $\mathrm{Wnt} / \beta$-catenin signaling pathway.

The Wnt/ $\beta$-catenin signaling pathway is the canonical Wnt signal pathway and was recently discovered and plays an important role in mediating the renal fibrosis. ${ }^{7,8}$ In unilateral ureteral obstruction rat models, all members of the Wnt family except Wnt5b, Wnt8b, and Wnt9b were upregulated in the fibrotic kidney with distinct dynamics. Numerous Wnt/ $\beta$-catenin target genes (c-Myc, Twist, lymphoid enhancerbinding factor 1 , and fibronectin) were induced. ${ }^{7}$ Oppositely, when administered Dkk1, a Wnt-pathway antagonist, could significantly reduce free $\beta$-catenin in cytoplasm and inhibit the downstream target gene transcription. ICG-001, a specific blocking $\beta$-catenin-mediated gene transduction, successfully blocked the expression of PAI-1, Col I, Fn, and $\alpha$-SMA induced by TGF- $\beta 1$ in HKC- 8 cells. In vivo study, ICG-001 could improve the renal fibrosis, inhibit overexpression of fiber-related protein, and reduce kidney damage. ${ }^{25}$

In this study, the $\mathrm{Wnt} / \beta$-catenin pathway was activated and Wnt1 was highly upregulated in 5/6 Nx models. Dkk1 as a upstream antagonist could bind to LRP5/6 receptor and induce LRP rapid endocytosis in participation of transmembrane protein Kremen1/2, which eventually inhibited the Wnt signal transduction into cytoplasm. ${ }^{26}$ The activity of Dkk1 was significantly high in the $5 / 6 \mathrm{Nx}$ group and in the HGF group was lower than it, which indicated that Dkk1 may possess a feedback regulation. The same characteristic was proved by using unilateral ureteral obstruction rat model, in which the expression of all the DKK family members increased remarkably. ${ }^{7,27}$ As the Wnt signal transduction into cell, $\beta$-catenin phosphorylation was then inhibited. So the level of $\beta$-catenin in the $5 / 6 \mathrm{Nx}$ group was high, and HGF could regulate it down. So the downstream target TCF4 was also inhibited in the HGF group. ${ }^{27,28}$ The Fn1, which was the main component of ECM and the target protein of Wnt pathway, was also decreased in the remnant kidney in HGF groups, which demonstrated that HGF could improve renal fibrosis by inhibiting the $\mathrm{Wnt} / \beta$-catenin pathway.

Wnt signaling pathway is silent within normal mature kidneys. ${ }^{29}$ However, a small amount of free $\beta$-catenin exists in the cytoplasm, and most of them combine with E-cadherin to form complexes in cell membrane, playing a crucial role in preventing cell migration and maintaining the same type of cell adhesion. ${ }^{30}$ While the Wnt pathway is activated, the degradation of free $\beta$-catenin is frustrated, leading to aggregation within the nucleus causing complexes to form with TCF families of LEF1/TCF, eventually accelerating the progression of epithelial-mesenchymal transition. ${ }^{31}$ Epithelialmesenchymal transition was the direct contributor to the kidney myofibroblast population and renal fibrosis. ${ }^{32,33}$ The GSK-3 $\beta$ mainly degrades $\beta$-catenin by forming degradation complex with adenomatous polyposis coli and Axin. It will be upregulated while the Wnt pathway is activated, ${ }^{34,35}$ which was also demonstrated in this study. However, HGF could not stimulate the expression of these two antagonists. Recent studies have shown that blockade of Wnt/ $\beta$-catenin signaling can simultaneously repress multiple renin-angiotensin system genes, which play an essential role in the pathogenesis of CKD and cardiovascular disease, ${ }^{36}$ and can also ameliorate proteinuria and kidney injury. ${ }^{37}$ So $\mathrm{Wnt} / \beta$-catenin signaling pathway becomes more and more important in the progress of CKD. Therefore, it can be used as the new therapeutic target for CKD.

\section{Conclusion}

In summary, the Wnt/ $\beta$-catenin signaling pathway has become more significant and may be a potential novel target for therapeutic intervention of CKD. The present study demonstrated that HGF improved remnant renal function and alleviated glomerulosclerosis and tubulointerstitial fibrosis potentially by depressing the Wnt/ $\beta$-catenin signaling pathway. In future studies, the spectrum-effect relationship of HGF will be identified, and the mechanism will be defined by gene silencing of $\mathrm{Wnt} / \beta$-catenin signaling pathway in vitro.

\section{Acknowledgments}

This work was supported by the Science and Technology Project of Guangdong, People's Republic of China (2011A030100007, 2011B031800128), the Natural Science Foundation of Guangdong, People's Republic of China 
(s2012010009056), and the Science and Technology Program of Guangzhou, People's Republic of China (11C32120715).

\section{Disclosure}

The authors declare no conflicts of interest in this work.

\section{References}

1. National Kidney Foundation. K/DOQI clinical practice guidelines for chronic kidney disease: evaluation, classification, and stratification. Am J Kidney Dis. 2002;39(2 suppl 1):S1-S266.

2. Jha $\mathrm{V}$, Garcia-Garcia $\mathrm{G}$, Iseki $\mathrm{K}$, et al. Chronic kidney disease: global dimension and perspectives. Lancet. 2013;382(9888):260-272.

3. Eddy AA. Overview of the cellular and molecular basis of kidney fibrosis. Kidney Int. 2014;4(1):2-8. [Suppl (2011)].

4. Duffield JS. Cellular and molecular mechanisms in kidney fibrosis. J Clin Invest. 2014;124(6):2299-2306.

5. Farris AB, Colvin RB. Renal interstitial fibrosis: mechanisms and evaluation. Curr Opin Nephrol Hypertens. 2012;21(3):289-300.

6. Lee SY, Kim SI, Choi ME. Therapeutic targets for treating fibrotic kidney diseases. Transl Res. 2015;165(4):512-530.

7. He W, Dai C, Li Y, Zeng G, Monga SP, Liu Y. Wnt/beta-catenin signaling promotes renal interstitial fibrosis. J Am Soc Nephrol. 2009;20(4): 765-776.

8. Nelson PJ, von Toerne C, Grone HJ. Wnt-signaling pathways in progressive renal fibrosis. Expert Opin Ther Targets. 2011;15(9): $1073-1083$.

9. Huang $\mathrm{H}, \mathrm{He} \mathrm{X}$. Wnt/beta-catenin signaling: new (and old) players and new insights. Curr Opin Cell Biol. 2008;20(2):119-125.

10. Mi K, Dolan PJ, Johnson GV. The low density lipoprotein receptorrelated protein 6 interacts with glycogen synthase kinase 3 and attenuates activity. J Biol Chem. 2006;281(8):4787-4794.

11. Feng JX, Hou FF, Liang M, et al. Restricted intake of dietary advanced glycation end products retards renal progression in the remnant kidney model. Kidney Int. 2007;71(9):901-911.

12. Tan X, He W, Liu Y. Combination therapy with paricalcitol and trandolapril reduces renal fibrosis in obstructive nephropathy. Kidney Int. 2009;76(12):1248-1257.

13. Zhang S, Wang D, Wang X, et al. Aqueous extract of Bai-Hu-Tang, a classical Chinese herb formula, prevents excessive immune response and liver injury induced by LPS in rabbits. J Ethnopharmacol. 2013; 149(1):321-327.

14. Wu N, Yang X, Song L, Wei J, Liu Z. Effect of Tianqi antitremor granules on behavioral manifestations and expression of $\mathrm{G}$ protein-coupled receptor kinase 6 and beta-arrestin1 in levodopa-induced dyskinesia in a rat model of Parkinson's disease. Drug Des Devel Ther. 2013;7: 1481-1489.

15. Fogo AB. Mechanisms of progression of chronic kidney disease. Pediatr Nephrol. 2007;22(12):2011-2022.

16. Tylicki L, Jakubowska A, Lizakowski S, Swietlik D, Rutkowski B. Management of renin-angiotensin system blockade in patients with chronic kidney disease under specialist care. Retrospective cross-sectional study. J Renin Angiotensin Aldosterone Syst. 2014;16(1):145-152.

17. Miao XH, Wang CG, Hu BQ, Li A, Chen CB, Song WQ. TGF-beta1 immunohistochemistry and promoter methylation in chronic renal failure rats treated with Uremic Clearance Granules. Folia Histochem Cytobiol. 2010;48(2):284-291.

18. Huang YR, Wei QX, Wan YG, et al. Ureic clearance granule, alleviates renal dysfunction and tubulointerstitial fibrosis by promoting extracellular matrix degradation in renal failure rats, compared with enalapril. J Ethnopharmacol. 2014;155(3):1541-1552.
19. Roberts MA. Commentary on the KDIGO clinical practice guideline for the management of blood pressure in chronic kidney disease. Nephrology (Carlton). 2014;19(1):53-55.

20. Wu JS, Liu Y, Shi R, Lu X, Ma YM, Cheng NN. Effects of combinations of Xiexin decoction constituents on diabetic nephropathy in rats. J Ethnopharmacol. 2014;157:126-133.

21. Zhang J, Wang P, Ouyang H, et al. Targeting cancer-related inflammation: Chinese herbal medicine inhibits epithelial-to-mesenchymal transition in pancreatic cancer. PLoS One. 2013;8(7):e70334.

22. Zhong Y, Deng Y, Chen Y, Chuang PY, Cijiang HJ. Therapeutic use of traditional Chinese herbal medications for chronic kidney diseases. Kidney Int. 2013;84(6):1108-1118.

23. Chan TM, Leung JK, Tsang RC, Liu ZH, Li LS, Yung S. Emodin ameliorates glucose-induced matrix synthesis in human peritoneal mesothelial cells. Kidney Int. 2003;64(2):519-533.

24. He D, Lee L, Yang J, Wang X. Preventive effects and mechanisms of rhein on renal interstitial fibrosis in obstructive nephropathy. Biol Pharm Bull. 2011;34(8):1219-1226.

25. Hao S, He W, Li Y, et al. Targeted inhibition of beta-catenin/CBP signaling ameliorates renal interstitial fibrosis. J Am Soc Nephrol. 2011;22(9): $1642-1653$.

26. González-Sancho JM, Aguilera O, García JM, et al. The Wnt antagonist DICKKOPF-1 gene is a downstream target of beta-catenin/TCF and is downregulated in human colon cancer. Oncogene. 2005;24(6): 1098-1103.

27. Banon-Maneus E, Rovira J, Ramirez-Bajo MJ, et al. Wnt pathway activation in long term remnant rat model. Biomed Res Int. 2014;2014: 324713.

28. Lu Z, Xie Y, Liu X, et al. Effect of 5/6 nephrectomized rat serum on epithelial-to-mesenchymal transition in vitro. Ren Fail. 2011;33(6): 600-608.

29. He X. Cilia put a brake on Wnt signalling. Nat Cell Biol. 2008;10(1): $11-13$.

30. Nelson WJ, Nusse R. Convergence of Wnt, beta-catenin, and cadherin pathways. Science. 2004;303(5663):1483-1487.

31. Medici D, Hay ED, Olsen BR. Snail and Slug promote epithelialmesenchymal transition through beta-catenin-T-cell factor-4-dependent expression of transforming growth factor-beta3. Mol Biol Cell. 2008;19(11):4875-4887.

32. Kriz W, Kaissling B, Le Hir M. Epithelial-mesenchymal transition (EMT) in kidney fibrosis: fact or fantasy? J Clin Invest. 2011;121(2):468-474.

33. Zhou D, Tan RJ, Zhou L, Li Y, Liu Y. Kidney tubular beta-catenin signaling controls interstitial fibroblast fate via epithelial-mesenchymal communication. Sci Rep. 2013;3:1878.

34. Surendran K, Schiavi S, Hruska KA. Wnt-dependent beta-catenin signaling is activated after unilateral ureteral obstruction, and recombinant secreted frizzled-related protein 4 alters the progression of renal fibrosis. J Am Soc Nephrol. 2005;16(8):2373-2384.

35. Gong R, Ge Y, Chen S, et al. Glycogen synthase kinase 3beta: a novel marker and modulator of inflammatory injury in chronic renal allograft disease. Am J Transplant. 2008;8(9):1852-1863.

36. Zhou L, Li Y, Hao S, et al. Multiple genes of the renin-angiotensin system are novel targets of Wnt/beta-catenin signaling. $J$ Am Soc Nephrol. 2015;26(1):107-120.

37. He W, Kang YS, Dai C, Liu Y. Blockade of Wnt/beta-catenin signaling by paricalcitol ameliorates proteinuria and kidney injury. J Am Soc Nephrol. 2011;22(1):90-103. 


\section{Supplementary materials}

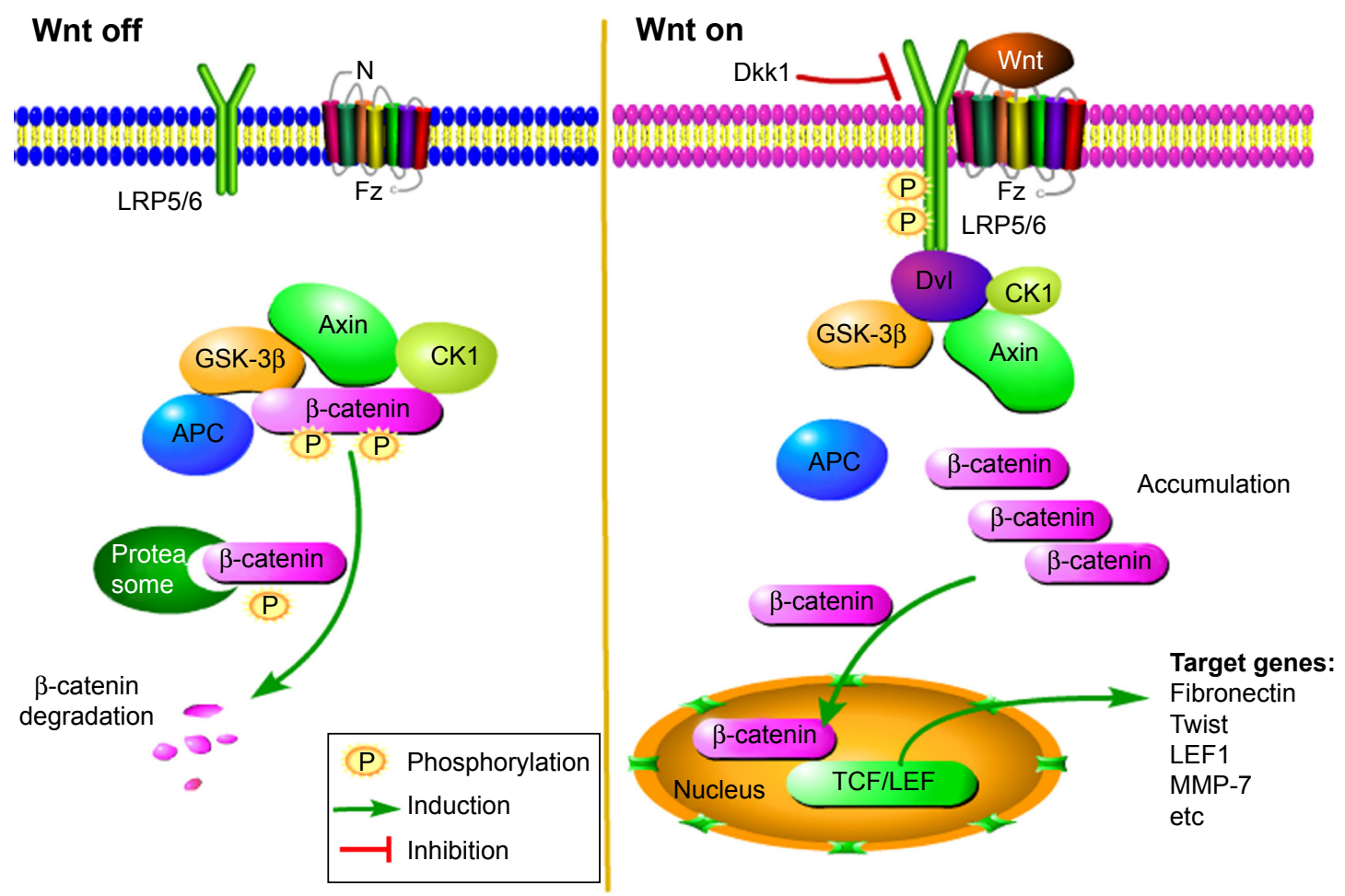

Figure SI Simplified schematic of the canonical Wnt/ $\beta$-catenin signaling pathway.

Notes: When Wnt is off, $\beta$-catenin is phosphorylated by a complex composed of APC, Axin, GSK-3 $\beta$, and CKI, which lead to the degradation of $\beta$-catenin via the proteasome pathway; When Wnt is on, Wnt ligands bind to and activate Fz and LRP5/6 and induce the phosphorylation of LRP6, which recruits Axin through Dvl to the plasma membrane resulting in the inhibition of $\beta$-catenin phosphorylation and degradation. Accumulated $\beta$-catenin translocates to the nucleus and complexes with the TCF/ LEF, moderating canonical Wnt-target gene transcription.

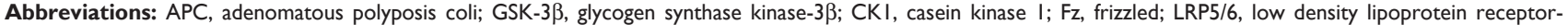
related protein 5 or 6; Dvl, disheveled; TCF/LEF, transcription factor/lymphoid enhancer factor; DkkI, dickkopf I; LEFI, lymphoid enhancer factor I; MMP-7, matrix metalloproteinase 7 .

Table SI Primers used for RT-PCR assay

\begin{tabular}{|c|c|c|c|c|}
\hline Gene ID & Gene symbol & Sequence $\left(5^{\prime}-3^{\prime}\right)$ & $\begin{array}{l}\text { Size of PCR } \\
\text { products (bp) }\end{array}$ & $\begin{array}{l}\text { Amplification } \\
\text { efficiency }\end{array}$ \\
\hline \multirow[t]{2}{*}{24881} & Wntl & Forward primer: CTTCGGCAAGATCGTCAACC & 51 & 101.05 \\
\hline & & Reverse primer: GCGAAGATGAACGCTGTTTCT & & \\
\hline \multirow[t]{2}{*}{84353} & $\beta$-catenin (Ctnnbl) & Forward primer: TGAAGGTGCTGTCTGTCTGCTC & 143 & 100.8 \\
\hline & & Reverse primer: TGCATCGGACAAGTTTCTCAGA & & \\
\hline \multirow[t]{2}{*}{84382} & TCF4 & Forward primer: CCAGTGGAATGAATCAGCCC & 190 & 98.9 \\
\hline & & Reverse primer: GAGGTGCTGTAATGGTTTGTGC & & \\
\hline \multirow[t]{2}{*}{84027} & GSK-3 $\beta$ & Forward primer: ATCTTTGGAGCCACCGATTACA & 160 & 98.6 \\
\hline & & Reverse primer: TTTGCTCCСTTGTTGGTGTTC & & \\
\hline \multirow[t]{2}{*}{293897} & Dkkl & Forward primer: CGAGGAAGGCATCATTGAAAAC & 143 & 100.3 \\
\hline & & Reverse primer: CTGTGGCGCAGTCTGATGATC & & \\
\hline \multirow[t]{2}{*}{25661} & $\mathrm{FnI}$ & Forward primer: TGACAACTGCCGTAGACCTGG & 72 & 99.7 \\
\hline & & Reverse primer: TACTGGTTGTAGGTGTGGCCG & & \\
\hline \multirow[t]{2}{*}{81822} & $\beta$-actin (Actb) & Forward primer: GGGAAATCGTGCGTGACATT & 76 & 105.7 \\
\hline & & Reverse primer: GCGGCAGTGGCCATCTC & & \\
\hline
\end{tabular}

Abbreviations: RT-PCR, reverse transcriptase-polymerase chain reaction; TCF4, transcription factor 4; GSK-3 $\beta$, glycogen synthase kinase-3 $\beta$; DkkI, dickkopf I; FnI, fibronectin I. 
Table S2 Relative retention time of the common peaks of ten batches of HGF extracts

\begin{tabular}{|c|c|c|c|c|c|c|c|c|c|c|}
\hline & Peak I & Peak 2 & Peak 3 & Peak 4 & Peak 5 & Peak 6 & Peak 7 & Peak 8 & Peak 9 & Peak 10 \\
\hline SI & 0.1370 & 0.1483 & 0.1691 & 0.1820 & 0.1915 & 0.2592 & 0.2683 & 0.3269 & 0.3731 & 0.3996 \\
\hline S2 & 0.1376 & 0.1485 & 0.1689 & 0.1852 & 0.2004 & 0.2604 & 0.2690 & 0.3314 & 0.3740 & 0.4011 \\
\hline S3 & 0.1379 & 0.1486 & 0.1692 & 0.1825 & 0.1997 & 0.2604 & 0.2692 & 0.3307 & 0.3745 & 0.4015 \\
\hline S4 & 0.1377 & 0.1482 & 0.1691 & 0.1823 & 0.1997 & 0.2605 & 0.2694 & 0.3303 & 0.3744 & 0.4015 \\
\hline S5 & 0.1378 & 0.1484 & 0.1692 & 0.1828 & 0.1998 & 0.2602 & 0.2693 & 0.3296 & 0.3745 & 0.4017 \\
\hline S6 & 0.1380 & 0.1484 & 0.1693 & 0.1824 & 0.1997 & 0.2604 & 0.2695 & 0.3291 & 0.3749 & 0.4020 \\
\hline S7 & 0.1380 & 0.1483 & 0.1693 & 0.1823 & 0.1917 & 0.2602 & 0.2695 & 0.3279 & 0.3749 & 0.4020 \\
\hline S8 & 0.1380 & 0.1483 & 0.1694 & 0.1820 & 0.1915 & 0.2603 & 0.2698 & 0.3268 & 0.3753 & 0.4024 \\
\hline S9 & 0.1378 & $0.148 \mid$ & 0.1694 & 0.1821 & 0.1908 & 0.2599 & 0.2695 & 0.3250 & 0.3749 & 0.4021 \\
\hline SIO & 0.1376 & 0.1484 & 0.1692 & 0.1778 & 0.1906 & 0.2575 & 0.2669 & 0.3235 & 0.3737 & 0.3970 \\
\hline
\end{tabular}

Abbreviation: HGF, Huang Gan formula.

Table S3 Relative areas of the common peaks of ten batches of extracts of HGF

\begin{tabular}{|c|c|c|c|c|c|c|c|c|c|c|}
\hline & Peak I & Peak 2 & Peak 3 & Peak 4 & Peak 5 & Peak 6 & Peak 7 & Peak 8 & Peak 9 & Peak 10 \\
\hline SI & 0.14 & 0.17 & 0.18 & 0.45 & 0.06 & 0.03 & 0.05 & 0.07 & 0.10 & 0.20 \\
\hline $\mathrm{S} 2$ & 0.19 & 0.28 & 0.17 & 0.43 & 0.17 & 0.05 & 0.05 & 0.07 & 0.11 & 0.22 \\
\hline S3 & 0.18 & 0.23 & 0.20 & 0.44 & 0.10 & 0.05 & 0.06 & 0.08 & 0.12 & 0.23 \\
\hline S4 & 0.17 & 0.17 & 0.18 & 0.51 & 0.12 & 0.03 & 0.04 & 0.07 & 0.10 & 0.20 \\
\hline S5 & 0.20 & 0.20 & 0.24 & 0.46 & 0.12 & 0.04 & 0.06 & 0.09 & 0.13 & 0.26 \\
\hline S6 & 0.21 & 0.20 & 0.26 & 0.59 & 0.07 & 0.04 & 0.06 & 0.07 & 0.11 & 0.23 \\
\hline S7 & 0.13 & 0.12 & 0.15 & 0.38 & 0.12 & 0.03 & 0.04 & 0.07 & 0.08 & 0.19 \\
\hline S8 & 0.22 & 0.17 & 0.21 & 0.48 & 0.14 & 0.03 & 0.05 & 0.07 & 0.09 & 0.21 \\
\hline S9 & 0.19 & 0.18 & 0.22 & 0.54 & 0.07 & 0.04 & 0.06 & 0.08 & 0.12 & 0.26 \\
\hline SIO & 0.17 & 0.18 & 0.27 & 0.40 & 0.07 & 0.03 & 0.07 & 0.06 & 0.09 & 0.21 \\
\hline
\end{tabular}

Abbreviation: HGF, Huang Gan formula.

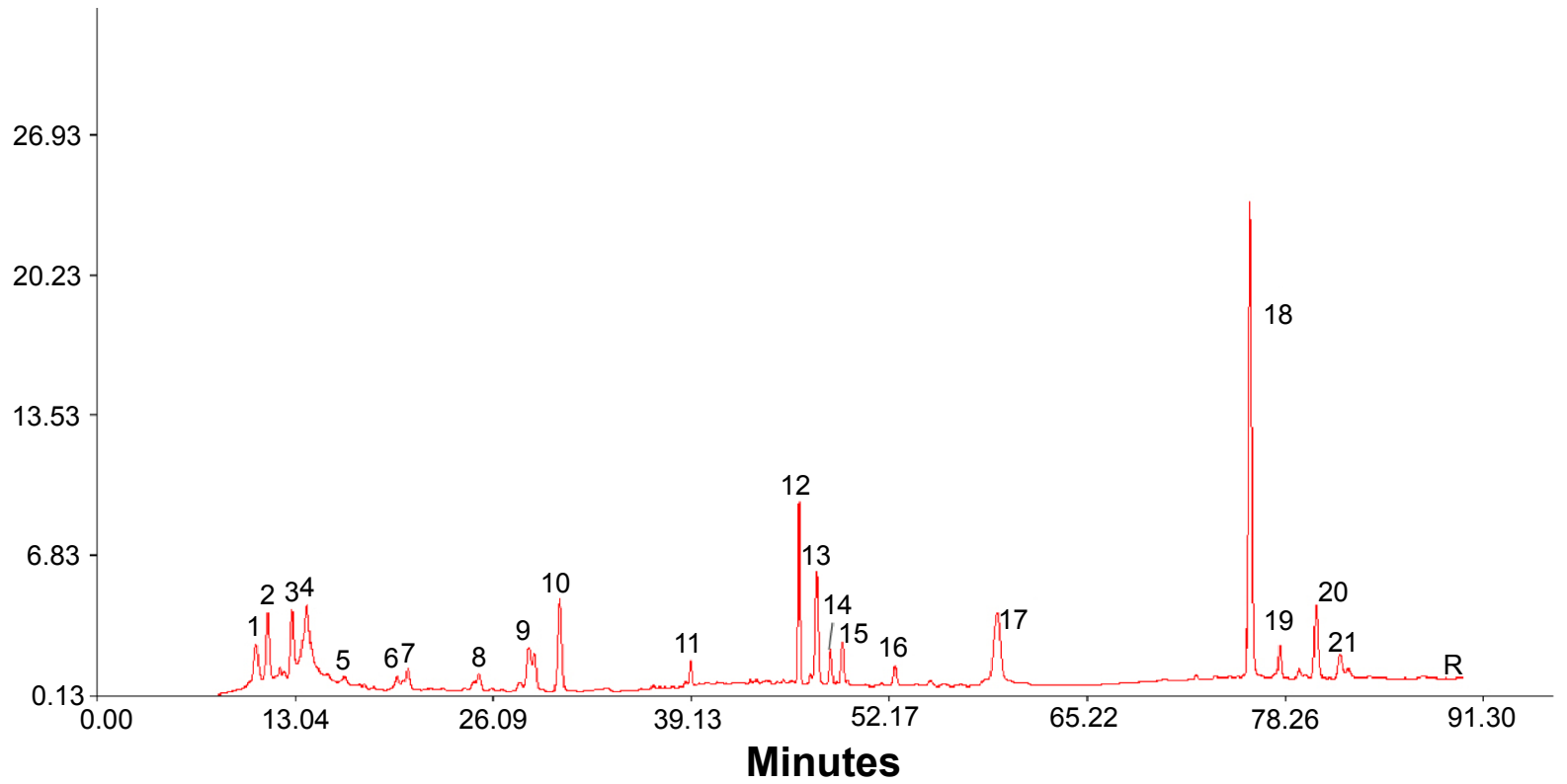

Figure S2 The reference fingerprint of HGF.

Notes: The reference fingerprint of HGF, which is produced according to HPLC chromatograms of the ten batches of HGF extracts by Similarity Evaluation System for Chromatographic Fingerprint of Traditional Chinese Medicine (Version 2004A). A total of 21 common peaks are marked, and the maximum peak 18 is set as the reference chromatographic peak, while the retention time and peak area are set to I. The relative retention time and relative peak area of common peaks compared to peak 18 are calculated. Fingerprint results showed that the ten batches of HGF possessed well consistency and the extraction method was stable. HPLC fingerprint can better control the overall quality of HGF extract.

Abbreviations: HGF, Huang Gan formula; HPLC, high-performance liquid chromatography. 


\begin{tabular}{|c|c|c|c|c|c|c|c|c|c|c|}
\hline Peak I I & Peak I 2 & Peak I3 & Peak I4 & Peak I5 & Peak 16 & Peak I7 & Peak I8 & Peak 19 & Peak 20 & Peak 21 \\
\hline 0.5119 & 0.6085 & 0.6232 & 0.6353 & $0.645 I$ & 0.6898 & 0.777 I & 1.0000 & 1.0259 & 1.0577 & $1.078 \mid$ \\
\hline 0.5143 & 0.6090 & 0.6238 & 0.6364 & 0.6462 & 0.6919 & 0.7831 & 1.0000 & 1.0257 & 1.0575 & $1.078 \mid$ \\
\hline 0.5144 & 0.6089 & 0.6239 & $0.636 I$ & 0.6460 & 0.6917 & 0.7826 & 1.0000 & 1.0256 & 1.0574 & 1.0780 \\
\hline 0.5146 & 0.6089 & 0.6239 & $0.636 I$ & $0.646 I$ & 0.6917 & 0.7818 & 1.0000 & 1.0256 & 1.0574 & 1.0780 \\
\hline 0.5145 & 0.6091 & 0.6240 & 0.6363 & 0.6463 & 0.6919 & 0.7806 & 1.0000 & 1.0256 & 1.0575 & 1.0782 \\
\hline 0.5146 & 0.6091 & 0.6240 & 0.6362 & 0.6462 & 0.6918 & 0.7797 & 1.0000 & 1.0256 & 1.0575 & 1.0782 \\
\hline 0.5147 & 0.6090 & $0.624 I$ & 0.6362 & 0.6462 & 0.6918 & 0.7788 & 1.0000 & 1.0257 & 1.0576 & 1.0782 \\
\hline 0.5150 & 0.6090 & 0.6240 & 0.6363 & 0.6463 & 0.6920 & 0.7775 & 1.0000 & 1.0256 & 1.0576 & 1.0782 \\
\hline 0.5148 & 0.6091 & $0.624 I$ & 0.6362 & 0.6462 & 0.6918 & 0.7757 & 1.0000 & 1.0256 & 1.0575 & 1.0783 \\
\hline 0.5123 & $0.607 \mid$ & 0.6224 & 0.6332 & 0.6428 & 0.6865 & 0.7759 & 1.0000 & 1.0262 & 1.0579 & 1.0788 \\
\hline
\end{tabular}

\begin{tabular}{lllllllllll}
\hline Peak I I & Peak I2 & Peak I3 & Peak I4 & Peak I5 & Peak I6 & Peak I7 & Peak I8 & Peak I9 & Peak 20 & Peak 2 I \\
\hline 0.05 & 0.19 & 0.24 & 0.05 & 0.07 & 0.04 & 0.35 & 1.00 & 0.06 & 0.19 & 0.07 \\
0.04 & 0.24 & 0.18 & 0.08 & 0.07 & 0.05 & 0.29 & 1.00 & 0.07 & 0.13 & 0.06 \\
0.05 & 0.34 & 0.25 & 0.10 & 0.12 & 0.07 & 0.40 & 1.00 & 0.07 & 0.19 & 0.05 \\
0.05 & 0.19 & 0.24 & 0.05 & 0.07 & 0.04 & 0.40 & 1.00 & 0.05 & 0.19 & 0.05 \\
0.05 & 0.20 & 0.19 & 0.06 & 0.07 & 0.04 & 0.27 & 1.00 & 0.08 & 0.12 & 0.08 \\
0.05 & 0.21 & 0.25 & 0.06 & 0.08 & 0.04 & 0.36 & 1.00 & 0.05 & 0.19 & 0.07 \\
0.03 & 0.13 & 0.20 & 0.03 & 0.05 & 0.03 & 0.38 & 1.00 & 0.03 & 0.18 & 0.05 \\
0.04 & 0.17 & 0.23 & 0.05 & 0.06 & 0.03 & 0.36 & 1.00 & 0.07 & 0.18 & 0.06 \\
0.05 & 0.19 & 0.25 & 0.06 & 0.07 & 0.04 & 0.39 & 1.00 & 0.09 & 0.18 & 0.07 \\
0.04 & 0.18 & 0.20 & 0.05 & 0.06 & 0.03 & 0.29 & 1.00 & 0.07 & 0.13 & 0.03 \\
\hline
\end{tabular}

Table S4 The MS qualitative analysis results of HGF (the mass spectrometry data of main eight ingredients)

\begin{tabular}{|c|c|c|c|c|c|c|c|c|}
\hline $\begin{array}{l}\text { Peak } \\
\text { number }\end{array}$ & $\begin{array}{l}\text { Retention } \\
\text { time (min) }\end{array}$ & Compound & $\begin{array}{l}\text { Molecular } \\
\text { formula }\end{array}$ & $\begin{array}{l}\text { Molecular } \\
\text { weight (MW) }\end{array}$ & $\begin{array}{l}\text { Measured } \\
\text { value }\end{array}$ & $\begin{array}{l}\text { Error value } \\
\text { (ppm) }\end{array}$ & $\begin{array}{l}\text { Matching } \\
\text { score }\end{array}$ & Adduction \\
\hline I & 10.633 & Liquiritin & $\mathrm{C}_{21} \mathrm{H}_{22} \mathrm{O}_{9}$ & 418.3940 & 417.1185 & -1.44 & 91.84 & {$[\mathrm{M}-\mathrm{H}]-$} \\
\hline 2 & 12.758 & Tectoridin & $\mathrm{C}_{22} \mathrm{H}_{22} \mathrm{O}_{11}$ & 462.4035 & 461.1089 & 0.00 & 96.79 & {$[\mathrm{M}-\mathrm{H}]-$} \\
\hline 3 & 39.25 & Luteolin & $\mathrm{C}_{15} \mathrm{H}_{10} \mathrm{O}_{6}$ & 286.23 & 285.0402 & -1.05 & 89.06 & {$[\mathrm{M}-\mathrm{H}]-$} \\
\hline 4 & 44.243 & Aloe-emodin & $\mathrm{C}_{15} \mathrm{H}_{10} \mathrm{O}_{5}$ & 270.23 & $271.365 I$ & 2.41 & 87.80 & {$[\mathrm{M}+\mathrm{H}]+$} \\
\hline 5 & 45.598 & Rhein & $\mathrm{C}_{15} \mathrm{H}_{8} \mathrm{O}_{6}$ & 284.22 & 283.0246 & -0.71 & 89.20 & {$[\mathrm{M}-\mathrm{H}]-$} \\
\hline 6 & 64.0553 & Emodin & $\mathrm{C}_{15} \mathrm{H}_{10} \mathrm{O}_{5}$ & 270.23 & 269.0447 & -2.97 & 92.08 & {$[\mathrm{M}-\mathrm{H}]-$} \\
\hline 7 & 74.158 & Chrysophanol & $\mathrm{C}_{15} \mathrm{H}_{10} \mathrm{O}_{4}$ & 254.23 & 253.0498 & -3.16 & 90.23 & {$[\mathrm{M}-\mathrm{H}]-$} \\
\hline 8 & 78.692 & Physcion & $\mathrm{C}_{16} \mathrm{H}_{12} \mathrm{O}_{5}$ & 284.27 & 283.0606 & -2.12 & 82.12 & {$[\mathrm{M}-\mathrm{H}]-$} \\
\hline
\end{tabular}

Abbreviations: MS, mass spectrometry; HGF, Huang Gan formula.

\section{Publish your work in this journal}

Drug Design, Development and Therapy is an international, peerreviewed open-access journal that spans the spectrum of drug design and development through to clinical applications. Clinical outcomes, patient safety, and programs for the development and effective, safe, and sustained use of medicines are a feature of the journal, which has also been accepted for indexing on PubMed Central. The manuscript management system is completely online and includes a very quick and fair peer-review system, which is all easy to use. Visit http://www.dovepress.com/testimonials.php to read real quotes from published authors. 